\title{
Gravitationally Focused Dark Matter Around Compact Stars
}

\author{
Benjamin C. Bromley \\ Department of Physics 83 Astronomy, University of Utah, \\ 115 S 1400 E, Rm 201, Salt Lake City, UT 84112 \\ bromley@physics.utah.edu
}

\begin{abstract}
If dark matter self-annihilates then it may produce an observable signal when its density is high. The details depend on the intrinsic properties of dark matter and how it clusters in space. For example, the density profile of some dark matter candidates may rise steeply enough toward the Galactic Center that selfannihilation may produce detectable $\gamma$-ray emission. Here, we discuss the possibility that an annihilation signal may arise near a compact object (e.g., neutron star or black hole) even when the density of dark matter in the neighborhood of the object is uniform. Gravitational focusing produces a local enhancement of density, with a profile that falls off approximately as the inverse square-root of distance from the compact star. While geometric dilution may overwhelm the annihilation signal from this local enhancement, magnetic fields tied to the compact object can increase the signal's contrast relative to the background.
\end{abstract}

\section{Introduction}

Dark matter accounts for the majority of the mass in the Universe, yet its identity remains elusive. Candidates include weakly interacting massive particles (WIMPs) like the neutralino $(\chi)$, the supersymmetric partner of the neutrino (Pagels \& Primack 1982), although their properties are only loosely constrained by theory and experiment. In some cases, plausible values of the mass and cross section suggest that self-annihilation signatures may be detectable in regions where the density of dark matter is high (Berezinsky et al. 1992; Bergström \& Gondolo 1996; Bertone et al. 2004). For example, Bergström et al. (1998) calculate the gamma ray flux from neutralino self-annihilation in the Galactic center (see also Zaharijas \& Hooper 2006), while Tyler (2002) and Bergström \& Hooper (2006) provide estimates of the annihilation signal from the nearby Draco dwarf galaxy. Intermediate-mass black holes may yield a WIMP annihilation signal (Bertone et al.|2005), as may remnant dark 
matter minihalos distributed throughout the Galaxy (e.g. Berezinsky et al. 2003; Sandick et al. 2011).

The observability of an annihilation signal critically depends on the number density of particles, since the local event rate is proportional to the density squared. Estimates of the strength of this signal typically derive from the assumption that dark matter density profiles follow a power law, $\rho \sim r^{-\gamma}$ in many astrophysical contexts. The power-law index $\gamma$ is between 1 and 2 in the central regions of galaxies according to cosmological simulations (Navarro et al. 1996; Moore et al. 1999; Power et al. 2003), corresponding to a density "cusp." If the simulations realistically describe the distribution of dark matter, then self-annihilation of WIMPS may indeed be observable in the centers of galaxies.

The presence of massive black holes in the centers of galaxies may further enhance the steep rise of a dark matter density profile. Gondolo \& Silk (1999) model the adiabatic growth of a central black hole to show that a density "spike," with a profile steeper than $r^{-2}$, forms around the black hole. Dynamical processes, such as scattering by stars and capture the black hole, may erode a spike over time, although it may remain largely intact (Bertone \& Merritt 2005). More problematic is the inspiral of smaller massive black holes captured from accreted galaxies that may disrupt a density spike (Merritt et al. 2002). Binary black hole coalescence can destroy density structures in the vicinity of the central black hole. However, even if merger event disrupts a density spike, relaxation processes may regenerate a dark matter "crest," with a density profile that falls off as $r^{-1.5}$ (Merritt et al. 2007). The time scale for the growth of a dark matter crest can be long, 10 Gyr in the case of the Milky Way, so that its structure could reflect the merger history of the Galaxy.

From an observational perspective, strong density cusps or spikes are not obviously common. For instance, Kravtsov et al. (1998) find that dwarf and low surface brightness galaxies have shallow core profiles, with $\gamma<0.5$, consistent with results from van den Bosch \& Swaters (2001) in a study of dwarf galaxies. Milosavljević et al. (2002) identify an anticorrelation between profile steepness and mass of the central black hole, lending support for a scenario in which the black hole grows through a sequence of merger events which tend to reduce or destroy any density cusp.

Even if no density cusp or spike exists in a galactic nucleus, the presence of a massive black hole, such as Sgr A* in the center of the Milky Way (Melia \& Falcke 2001), can facilitate annihilation radiation. The reason is that gravitational focusing inevitably leads to a dark matter density enhancement near the black hole. This phenomenon will occur for any star in a field of dark matter (Danby \& Camm 1957; Griest 1988; Sikivie \& Wick 2002; Alenazi $\&$ Gondolo 2006). The purpose of this paper is to review the gravitational focusing effect and discuss its observational consequences in terms of dark matter annihilation radiation. 


\section{Gravitational focusing}

A point particle with mass $M$ in a uniform bath of hot, dissipationless dark matter will influence dark matter orbits, but in a manner which conserves phase-space density along each trajectory. Danby \& Camm (1957) derived analytical expressions for the density enhancement near the point mass in a bath of unbound particles with a Maxwellian velocity distribution with velocity dispersion $\sigma_{v}$. For a point mass which is at rest with respect to the bulk dark matter, the density enchancement is

$$
\frac{\rho}{\rho_{\infty}}=\sqrt{\frac{2}{\pi}} q+e^{q^{2} / 2}[1-\operatorname{erf}(q / \sqrt{2})],
$$

where $q=\sqrt{2 G M / \sigma_{v}^{2} r}$ is the ratio of escape velocity at distance $r$ from the point mass. Thus, close to the point mass the density enhancement grows with decreasing radius according to $r^{-0.5}$. Note that details of Dandy \& Camm's results have been contested by Griest (1988), and more recently by Sikivie \& Wick (2002). The final word comes from Alenazi \& Gondolo (2006), who resolve the discrepancies.

To support the previous analytical work, and to provide a framework for studying gravitational focusing about an arbitrary mass distribution, I developed a computer code to track the flow of dark matter about a compact star. The code calculates Monte Carlo orbits assuming that the dark matter has a Maxwellian velocity distribution at large distance from the star, and that the star may have some finite speed relative to the average rest frame of the dark matter. The resulting simulations show that the density enhancement from gravitational focusing can exceed two orders of magnitude near the surface of a neutron star, and even higher within a factor of a few times the Schwarzschild radius of a black hole. Here, to account for the possibility that a dark matter particle may be captured by the black hole, the code does not track orbits inside of five times the Schwarzschild radius (see Gondolo \& Silk 1999). The measure of particle trajectories that reach inside this distance is small, and numerical simulations demonstrate that these orbits do not greatly affect the density enhancement at larger radii. Thus analytical calculations for point particles are broadly applicable to compact objects.

Figure 1 illustrates the density enhancement at the Galactic center for a background dark matter halo distribution similar to one calculated by Merritt et al. (2002). The background model has a profile that falls off as $r^{-1.0}$, as predicted by numerical simulations (Navarro et al. 1996). A "core" has been imposed to crudely mimic the effects of dark matter clearing by black hole mergers of Merritt et al. (2002). To calculate the effect of gravitational focusing by Sgr A*, a black hole mass of $3 \times 10^{6} \mathrm{M}_{\odot}$ is assumed, and the velocity distribution is assigned to be isotropic with a dispersion of $\sigma_{v}=155 \mathrm{~km} / \mathrm{s}$. The black hole mass, the assumption 
of an isotropic velocity distribution, and the value of $\sigma_{v}$ used here are all approximately consistent with observed stellar kinematics in the Galactic center (Ghez et al. 1998; Genzel et al. 2000).

The observed dark matter self-annihilation flux depend on the local emissivity,

$$
j=\frac{Y\left\langle\sigma_{a n n} v\right\rangle \rho_{\mathrm{dm}}^{2}}{4 \pi m_{\mathrm{dm}}^{2}}
$$

where $\rho_{\mathrm{dm}} / m_{\mathrm{dm}}$ is the dark matter number density, and $\left\langle\sigma_{a n n} v\right\rangle$ gives the self-annihilation rate per unit density. For the neutralino, typical values from the literature (e.g. Bergström \& Gondolo 1996) are $m_{\chi} \equiv m_{\mathrm{dm}}=100 \mathrm{GeV}$, and $\left\langle\sigma_{a n n} v\right\rangle=10^{-26} \mathrm{~cm}^{3} / \mathrm{s}$, independent of pairwise closing speed $v$. The quantity $Y$ specifies the yield of decay by-products; for example, the bolometric yield corresponds to $Y=m_{\chi} c^{2}$. The emissivity per unit frequency of photons produced by electrons in a magnetic field depends on electron-positron production channels, as well as synchrotron radiative efficiencies Tyler (e.g., 2002).

In the case of certain neutralino decay products, namely neutrinos, the flux is a straightforward line-of-sight integral over the emissivity, since self-absorption and diffusion do not occur (Gondolo \& Silk 1999). The line of sight integral along some sky direction $\hat{n}$ is conveniently expressed in dimensionless form as (Bergström et al. 1998; Merritt et al. 2002)

$$
J(\hat{n})=\frac{1}{8.5 \mathrm{kpc}}\left(\frac{1}{0.3 \mathrm{GeV} / \mathrm{cm}^{3}}\right)^{2} \int_{\hat{n}} d \ell \rho_{\mathrm{dm}}^{2} .
$$

Figure 2 gives $J$, averaged inside a circular aperture centered on $\mathrm{Sgr} A *$, as a function of aperture radius. The density profile is the same as in Figure 1. The enhancement from gravitational focusing is significant inside small apertures. Even so, Bertone et al. (2004) point out that the neutrino flux from the Galactic Center will be undetectable if the current gamma-ray constraints are any indication of the annihilation rate. Erkoca et al. (2010) are more hopeful from a theoretical perspective, while the observations are providing limits to the neutrino flux (e.g., from IceCube Abbasi et al. 2011), but no Galactic Center signal at this point.

\section{Implications}

The strength of a gravitationally focused density profile around a compact object is unfortunately insufficient to generally produce a strong flux enhancement above that from the background density. In a uniform bath of dark matter the flux only grows logarithmically

with decreasing radius, and is much weaker than in the case of the density spike envisioned by 
Gondolo \& Silk (1999). Still, gravitational focusing may have astrophysical relevance if the flux is enhanced by any distinctive properties of the environment around the compact object. This situation may occur in the case of synchrotron radiation from a region around a black hole or neutron star. For supermassive black holes, magnetic field strengths can be orders of magnitude higher than in the interstellar medium. Just as the gravitational focusing is pinned to the black hole, so is the magnetic field, as it is likely tied to gas accreting on to the black hole.

An estimate of the synchrotron flux from Sgr A* follows from the density profile of Figure 1 and an equipartition model for the magnetic field strength (Melia 1992), in which $B=(r / p c)^{-1.2} \mathrm{mG}$. The value for electron-positron yield may be obtained as in Tyler (2002); in the low energy limit, the electron energy distribution per neutralino annihilation is $d N / d E \approx E^{-1.5}$. An integration of the radiative transfer equation should include the possible effects of synchrotron self-absorption. Synchrotron limits have been used by Bertone et al. (2001) to place constraints on dark matter properties. However, these limits depend on a strong flux generated in a density cusp and spike, along with a relatively strong magnetic field, all of which must be centered on Sgr A*. According to the results of Merritt et al. (2002), even a relatively minor merger between the Milky Way and a small galaxy can greatly reduce any annihilation signal. For example, if the Milky Way were to accrete a galaxy with a black hole whose mass is one tenth of Sgr A*, then a strong density enhancement near Sgr A* would be destroyed.

On the other hand, the more modest density peak produced by gravitational focusing will persist, even after a merger event, presuming that dark matter particle orbits in the vicinity of Sgr A* are randomized. Gravitational focusing will also occur around other, smaller compact objects in Galaxy. Consider, for example, a neutron star moving with considerable velocity through a thermal bath of dark matter. Figure 3 shows the density enhancement along the star's direction of motion, and Figure 4 illustrates the intensity of annihilation radiation from material in a plane containing the path of the star. The signal is strongest behind the star, and weaker in front, but the angle-averaged signal strength is not greatly different from the case where the star is at rest in the bulk dark matter frame.

A neutron star is an improbable source of annihilation radiation in the form of synchrotron photons; the volume where there is significant gravitational focusing is too small to be observable in the case of the neutralino. The physics is nonetheless remarkable. Neutralino self-annihilation through a charged pion channel in a $10^{12} \mathrm{G}$ magnetic field will produce a pion synchrotron signal, since the synchrotron cooling rate is fast $\left(<10^{-11} \mathrm{~s}\right)$ compared with the pion lifetime $\left(>10^{-8} \mathrm{~s}\right)$. Furthermore, quantum effects (Baring 1989) will be important in the case of a magnetar, with field strength in excess of $10^{14} \mathrm{G}$. Then, the emitted photons 
will have energies comparable to the typical pion energy.

\section{Conclusion}

Gravitational focusing will generally enhance the annihilation signal around any massive compact object. However, because of geometrical dilution, this signal may not be distinguishable from the background annihilation radiation. Fortunately, local conditions around a compact object may cause the annihilation signal to appear in wavebands which are different from the background. For example, magnetic fields tied to compact objects can generate a distinctive synchrotron radiation. Of the sources considered here-neutron stars, stellarmass black holes, and massive black holes - only the latter seems capable of producing a detectable signal by gravitational focusing alone. Neutron stars, specifically magnetars, may be the weakest sources, but they still promise interesting physics involving WIMP decay products.

Theoretical models of WIMP annihilation in the Galactic Center, bolstered by recent

observations with the Fermi Gamma-ray Space Telescope (e.g., Abdo et al. 2010), have the dark matter concentrated about Sgr A* as a result of uncertain galactic evolution and black hole growth dynamics. The main point of this work is that gravitational focusing will generate some signal, no matter what the formation history. While distinctive gamma ray signal could be lost in a uniform bath of WIMPs, synchrotron radiation may retain some contrast with the background. The trade-off with synchrotron signal is that the accretion flows that presumably generate the localized magnetic field may contaminate the signal. Our best hope may be to find isolated intermediate mass black holes (Bertone et al. 2005). Alternatively we might seek an underluminous active galactic nucleus. Fortunately, our own Galaxy has one.

\section{REFERENCES}

Abbasi, R., Abdou, Y., Abu-Zayyad, T., et al. 2011, Phys. Rev. D, 84, 022004

Abdo, A. A., et al. 2010, ApJS, 188, 405

Alenazi, M. S., \& Gondolo, P. 2006, Phys. Rev. D, 74, 083518

Baring, M. G. 1989, A\&A, 225, 260

Berezinsky, V., Dokuchaev, V., \& Eroshenko, Y. 2003, Phys. Rev. D, 68, 103003 
dm annihilation from small clumps as in cdm simulations

Berezinsky, V. S., Gurevich, A. V., \& Zybin, K. P. 1992, NASA STI/Recon Technical Report N, 93,28473

Bergström, L., \& Gondolo, P. 1996, Astroparticle Physics, 5, 263

Bergström, L., \& Hooper, D. 2006, Phys. Rev. D, 73, 063510

Bergström, L., Ullio, P., \& Buckley, J. H. 1998, Astroparticle Physics, 9, 137

Bertone, G., Hooper, D., \& Silk, J. 2004, Phys. Rep., 405, 279

Bertone, G., \& Merritt, D. 2005, Phys. Rev. D, 72, 103502

Bertone, G., Nezri, E., Orloff, J., \& Silk, J. 2004, Phys. Rev. D, 70, 063503

Bertone, G., Sigl, G., \& Silk, J. 2001, MNRAS, 326, 799

Bertone, G., Zentner, A. R., \& Silk, J. 2005, Phys. Rev. D, 72, 103517

Danby, J. M. A., \& Camm, G. L. 1957, MNRAS, 117, 50

Erkoca, A. E., Reno, M. H., \& Sarcevic, I. 2010, Phys. Rev. D, 82, 113006

Genzel, R., Pichon, C., Eckart, A., Gerhard, O. E., \& Ott, T. 2000, MNRAS, 317, 348

Ghez, A. M., Klein, B. L., Morris, M., \& Becklin, E. E. 1998, ApJ, 509, 678

Griest, K. 1988, Phys. Rev. D, 37, 2703

Gondolo, P., \& Silk, J. 1999, Physical Review Letters, 83, 1719

Kravtsov, A. V., Klypin, A. A., Bullock, J. S., \& Primack, J. R. 1998, ApJ, 502, 48

Melia, F. 1992, ApJ, 387, L25

Melia, F., \& Falcke, H. 2001, ARA\&A, 39, 309

Merritt, D., Harfst, S., \& Bertone, G. 2007, Phys. Rev. D, 75, 043517

Merritt, D., Milosavljević, M., Verde, L., \& Jimenez, R. 2002, Physical Review Letters, 88, 191301

Milosavljević, M., Merritt, D., Rest, A., \& van den Bosch, F. C. 2002, MNRAS, 331, L51

Moore, B., Quinn, T., Governato, F., Stadel, J., \& Lake, G. 1999, MNRAS, 310, 1147 
Navarro, J. F., Frenk, C. S., \& White, S. D. M. 1996, ApJ, 462, 563

Power, C., Navarro, J. F., Jenkins, A., Frenk, C. S., White, S. D. M., Springel, V., Stadel, J., \& Quinn, T. 2003, MNRAS, 338, 14

Pagels, H., \& Primack, J. R. 1982, Physical Review Letters, 48, 223

Sandick, P., Diemand, J., Freese, K., \& Spolyar, D. 2011, arXiv:1108.3820

Sikivie, P., \& Wick, S. 2002, Phys. Rev. D, 66, 023504

Tyler, C. 2002, Phys. Rev. D, 66, 023509

van den Bosch, F. C., \& Swaters, R. A. 2001, MNRAS, 325, 1017

Zaharijas, G., \& Hooper, D. 2006, Phys. Rev. D, 73, 103501 


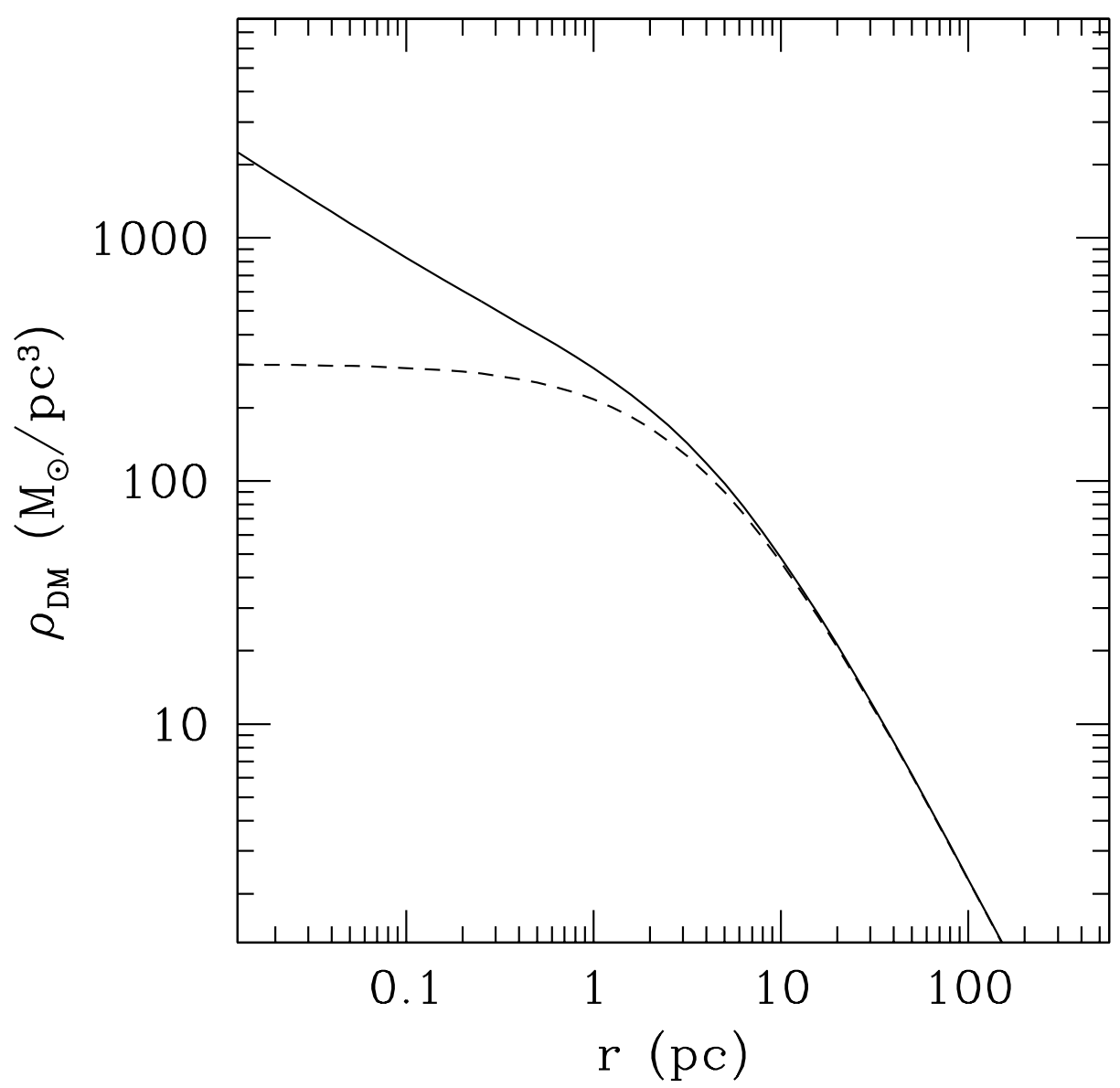

Fig. 1. - The density enhancement from gravitational focusing of dark matter in a density well that approximates the smoothing effect of mergers. The dashed line represents the dark matter without focusing, while the solid curve approximates the gravitationally focused profile. 


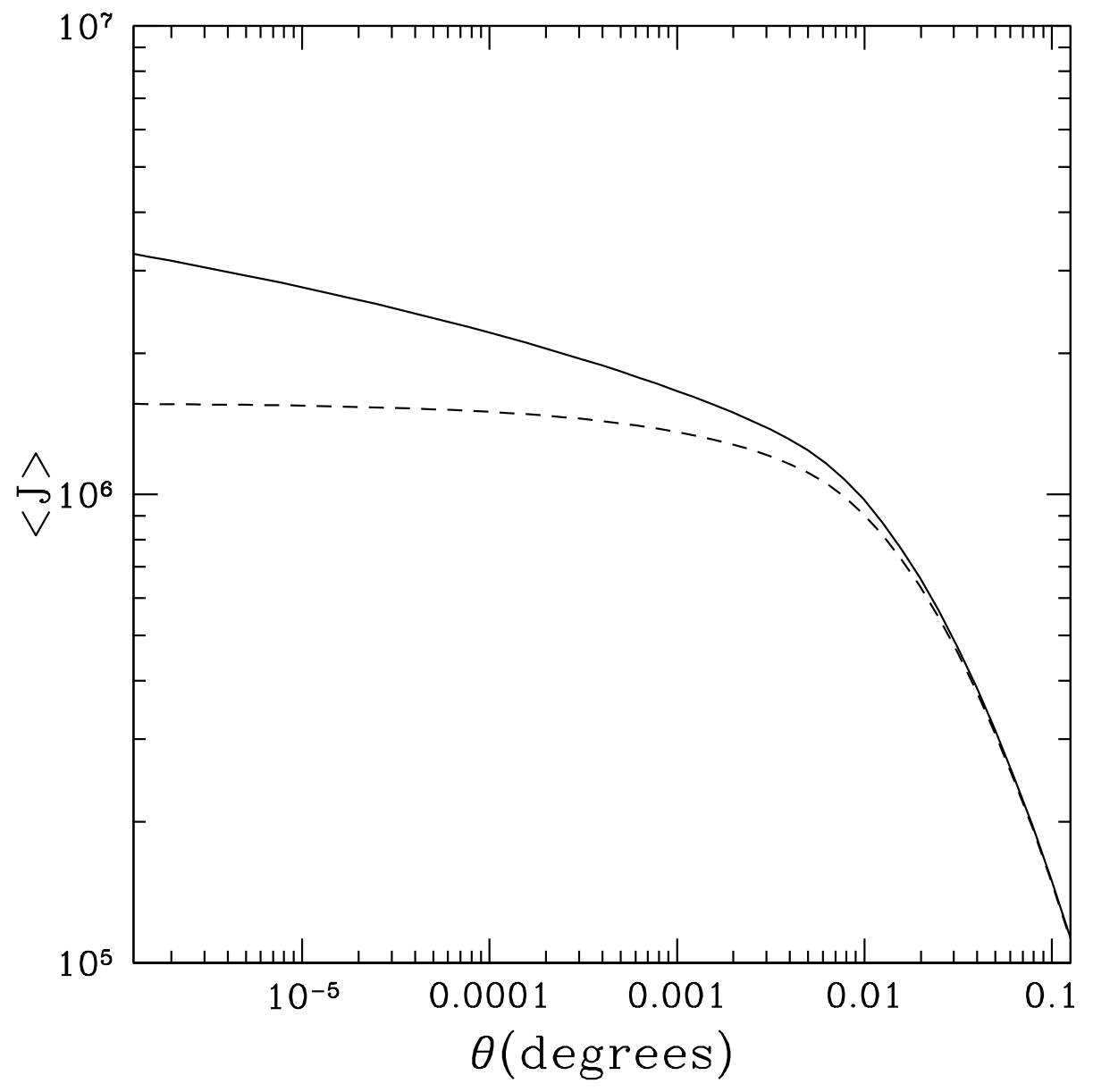

Fig. 2.- The dimensionless neutralino self-annihilation flux $J$ (eq. 3), averaged inside a circular beam of radius $\theta$, centered on Sgr A*. As in Fig. 1, the dashed line represents a calculation without the effect of gravitational focusing, while the solid curve includes the focusing effect. 


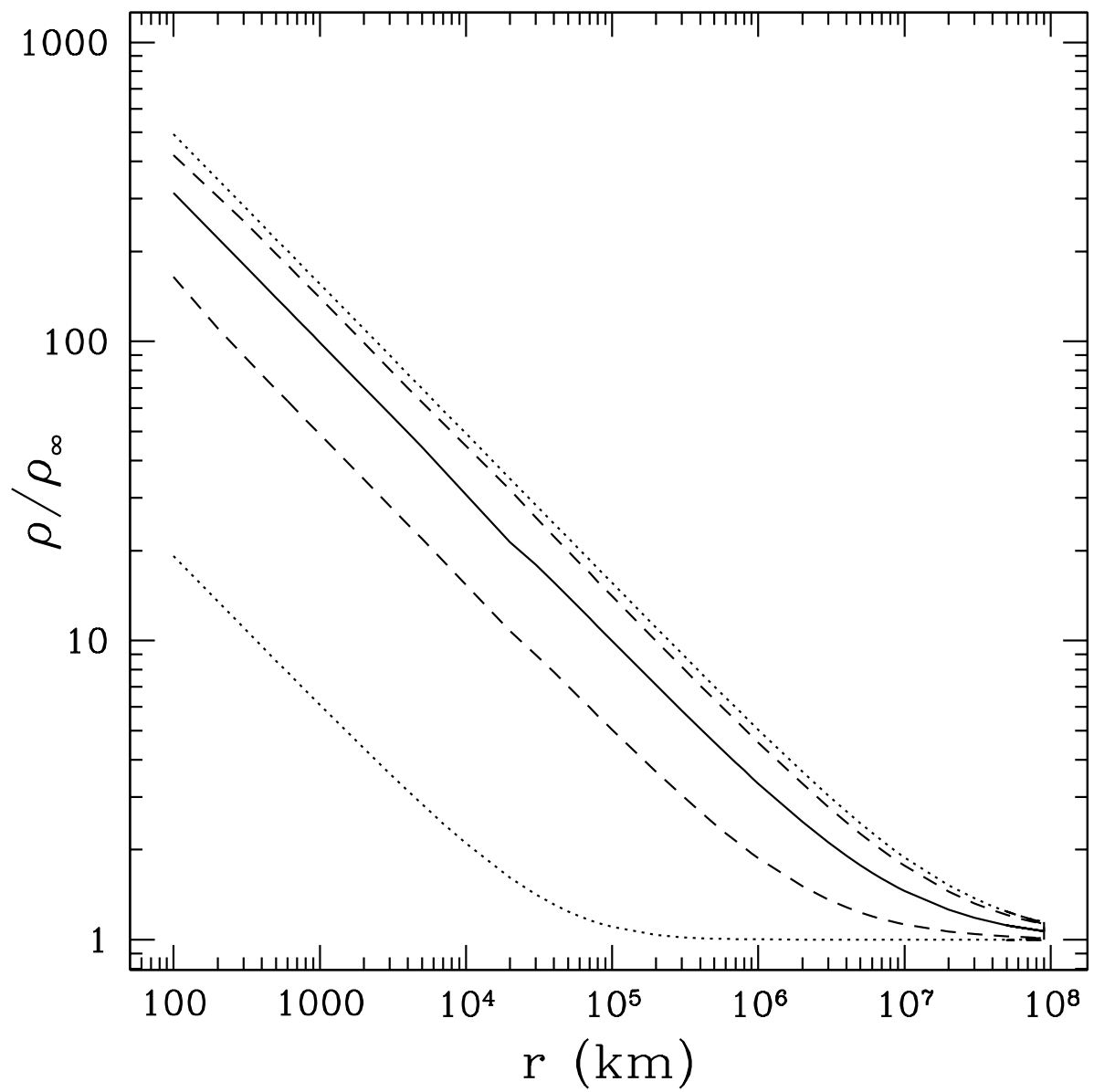

Fig. 3.- The density enhancement of thermal dark matter $\left(\sigma_{v}=155 \mathrm{~km} / \mathrm{s}\right)$ in the wake of a neutron star. The solid line shows the case where the neutron star is at rest with respect to the frame of the dark matter bath; the dashed and dotted lines correspond to neutron star velocities of $200 \mathrm{~km} / \mathrm{s}$ and $1600 \mathrm{~km} / \mathrm{s}$, respectively. The upper curves show focusing of the downstream flow, lower curves correspond to upstream density enhancement. In the limit of infinite star velocity, a downstream density caustic will emerge (Sikivie \& Wick 2002). 


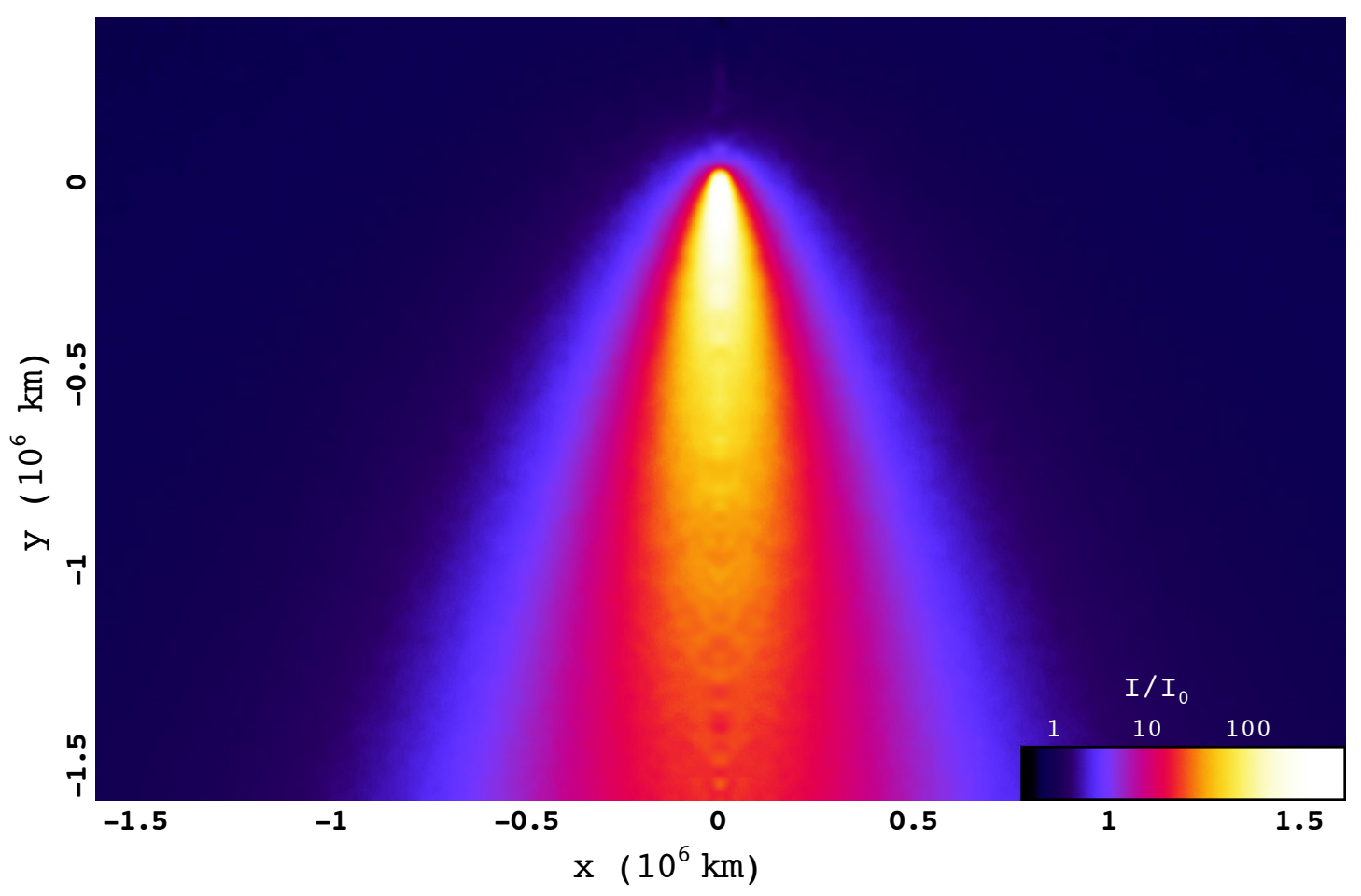

Fig. 4.- The intensity of self-annihilation radiation a plane containing the path of a highvelocity neutron star. In this instance, the neutron star (located at the origin) is moving at $1000 \mathrm{~km} / \mathrm{s}$ in a thermal bath of dark matter $\left(\sigma_{v}=200 \mathrm{~km} / \mathrm{s}\right)$. The color scale (see inset) corresponds to the $\log$ of the intensity $I$ (relative to the background signal $I_{0}$ ) from dark matter in a thin sheet containing this plane, as approxmated from a direct simulation of $\sim 10^{9}$ test particles passing by the star. 